Review

\title{
Immunoproteasome in IgA Nephropathy: State-of-Art and Future Perspectives
}

\author{
Ting Gan, Yang Li, Xu-jie Zhou ${ }^{\bowtie}$ and Hong Zhang \\ Renal Division, Peking University First Hospital; Peking University Institute of Nephrology; Key Laboratory of Renal Disease, Ministry of Health of China; Key \\ Laboratory of Chronic Kidney Disease Prevention and Treatment (Peking University), Ministry of Education, Beijing, 100034, People's Republic of China; \\ Research Units of Diagnosis and Treatment of Immune-mediated Kidney Diseases, Chinese Academy of Medical Sciences. \\ $\square$ Corresponding author: Dr. Xu-jie Zhou, MD \& PhD. E-mail: zhouxujie@bjmu.edu.cn; Renal Division, Peking University First Hospital, Peking University \\ Institute of Nephrology, Key Laboratory of Renal Disease, Ministry of Health of China; Key Laboratory of Chronic Kidney Disease Prevention and Treatment \\ (Peking University), Ministry of Education, Beijing, People's Republic of China. No. 8, Xishiku Street, Xicheng District, Beijing 100034, P.R China. Tel.: +86 10 \\ 83572388; Fax: +8610 66551055
}

(c) The author(s). This is an open access article distributed under the terms of the Creative Commons Attribution License (https://creativecommons.org/licenses/by/4.0/). See http://ivyspring.com/terms for full terms and conditions.

Received: 2020.05.18; Accepted: 2020.07.10; Published: 2020.07.19

\begin{abstract}
$\lg$ A nephropathy $(\operatorname{IgAN})$ is a leading cause of chronic kidney disease and renal failure. The exact pathogenesis of IgAN is not well defined, but some genetic studies have led to a novel discovery that the immunoproteasome probably plays an important role in IgAN. The immunoproteasome is a proteasome variant that is expressed when cells are stressed or receive inflammatory signals. While immunoproteasome is suggested to be mainly involved in major histocompatibility complex-I (MHC-I) antigen presentation, recent studies indicate that it may assert broad functions in trafficking events that activate both innate and adaptive immunity. In this review, we first summarize new insights into its functions in immunity, and discuss how it underlies its associations with $\lg \mathrm{AN}$. We also highlight its potential as a therapeutic target for the future.
\end{abstract}

Key words: IgA nephropathy; Glomerulonephritis; Immunoproteasome; Autoimmunity

\section{Introduction}

IgA nephropathy (IgAN), the most common primary glomerulonephritis globally, is a leading cause of chronic kidney disease (CKD) and end stage kidney disease (ESKD) [1]. Although the exact pathogenesis of IgAN is not well defined, a 'multi-hit' hypothesis has been proposed to explain the pathogenesis of IgAN. Specifically, the level of IgA1 bearing galactose-deficient O-glycans (Gd-IgA1) is increased in the circulation of patients with IgAN. These Gd-IgA1 are recognized as autoantigens by antiglycan autoantibodies, leading to the formation of immune complexes that accumulate in the glomerular mesangium [2]. These immune complexes activate mesangial cells, induce proliferation and promote the production of extracellular matrix, cytokines and chemokines, leading to renal injury [3]. However, this hypothesis does not explain the pathogenic mechanism of secondary IgAN. In addition, the etiologic factors in each hit remain largely unanswered. Newer insights arising from clinical and genetic studies contribute to the refinement of this hypothesis and suggest a genetic component to IgAN pathogenesis. It has been reported the familial aggregation of $\operatorname{IgAN}$ and the presence of Gd-IgA1 as a heritable trait [4]. With significant improvements in genotyping efficiency and sequencing technology in recent years, several large genome-wide association studies (GWAS) for IgAN have been performed[4-8], providing important insights into the potential mechanisms and pathways that contribute to the disease risk.

In one of the first large-scale GWAS conducted in IgAN, Gharavi, et al. [4] identified single nucleotide polymorphisms (SNP) located in the PSMB8 locus (rs2071543, a Q49K missense variant) associated with the susceptibility to IgAN. The PSMB8 locus encodes low molecular mass polypeptide 7 (LMP7) of the immunoproteasome, which is involved in antigen 
processing and presentation during infection. The typical exacerbation of hematuria in some patients following a mucosal infection suggests the participation of infectious agents in disease pathogenesis [9]. In agreement with this notion, Coppo, et al. [10] reported an upregulation of the immunoproteasome in peripheral blood mononuclear cells (PBMC) of patients with IgAN. Furthermore, they observed a trend for an increased proteasomeimmunoproteasome switch in patients with IgAN. It is of interest that the proteasome-immunoproteasome switch found in patients with IgAN was completely different from that of control subjects with respiratory or gastrointestinal infections without renal disease [9]. And in a more recent study, the correlation between LMP7/ $\beta 5$ switch in peripheral white blood cells and velocity of progression of IgAN was reported, and high LMP7/ $\beta 5$ switch may represent a biomarker for identifying patients at risk of progression [11]. These findings highlight the pathogenic significance of the proteasomal switch in IgAN.

In a shared genetics study between IgAN and systemic lupus erythematosus (SLE), we previously observed variants of UBE2L3, a gene involved in ubiquitin/proteasome pathway, were associated with susceptibility to both autoimmune diseases [12]. UBE2L3 has been further functionally validated involved in SLE by amplifying nuclear factor kappa B (NF-kB) activation and promoting plasma cell development [13]. In a single-center open-label pilot trial testing the effect of bortezomib (a proteasome inhibitor that targets proteasome subunits $\beta 5, \beta 5 \mathrm{i}$ and $\beta 1 i)$ in patients with severe IgAN and significant proteinuria. Different to rituximab in other autoimmune disease, B-cell depletion in $\operatorname{IgA}$ nephropathy, which has been suggested to be an autoimmune disease, did not show expected efficacy [14]. In comparison, after a 1-year follow-up in the trial, of all 8 subjects, 3 subjects ( $38 \%$ ) had complete remission with proteinuria less than $300 \mathrm{mg}$ per day [15]. The evidence suggests that the (immuno) proteasome may play an important role in IgAN. And it supported that long term remission could be achieved without corticosteroid treatment but just by a short duration of bortezomib. In this review, we summarize the latest progress on (immuno) proteasome, discuss potential mechanisms of the (immuno) proteasome involved in the pathogenesis of IgAN, and suggest future research directions focused on (immuno) proteasome.

\section{Structure and activity of the (immuno) proteasome}

The $26 \mathrm{~S}$ proteasome, the most common proteasome form in eukaryotic cells, is part of the ubiquitin-proteasome system (UPS) that is responsible for degrading damaged or misfolded proteins tagged with ubiquitin to maintain cellular homeostasis [16] (Fig. 1). The proteasome consists of two parts including 195 regulatory particles and a 205 catalytic core particle. The $20 \mathrm{~S}$ proteasome is composed of four heptameric rings with a barrel-shaped structure. Seven distinct a-subunits (a1- $\alpha 7)$ form the two outer a-rings and the two inner rings possess seven different $\beta$-subunits $(\beta 1-\beta 7)$ [17]. The proteolytic activity resides in $\beta 1, \beta 2$, and $\beta 5$ subunits, which are responsible for the caspase-like (C-L), trypsin-like (T-L), and chymotrypsin-like (ChT-L) activities, respectively [18]. Under the stimuli of certain pro-inflammatory cytokines such as interferon (IFN) $-\gamma$ and tumor necrosis factor (TNF)- $\alpha$, the $\beta 1, \beta 2$, and $\beta 5$ subunits are replaced by functionally different counterparts named $\beta 1$ i (low molecular mass polypeptide 2, LMP2), $\beta 2 \mathrm{i}$ (multicatalytic endopeptidase complex-like-1, MECL-1) and $\beta 5 \mathrm{i}$ (LMP7), forming the variant immunoproteasome [19]. The immunoproteasome is predominantly expressed in lymphocytes and monocytes of jawed vertebrates [20]. The changes in active site subunits cause an altered cleavage site preference as well as a different cleavage rate of immunoproteasome. In detail, the immunoproteasome has increased trypsinlike (T-L), and chymotrypsin-like (ChT-L) activities that are favorable for the production of antigenic peptides that bind to the groove of MHC-I [21]. In fact, there are several hybrid immunoproteasomes with only one or two of the three immunoproteasome subunits integrated. Each hybrid proteasome has a unique structural composition as well as specialized and diversified enzymatic properties to produce a specific repertoire of peptides, thus regulates a host of cellular processes [22].

\section{Antigen presentation by immunoproteasome}

From a clinical point of view, about $30 \%$ of patients with IgAN (mostly young subjects) have a clear history of disease exacerbation after upper respiratory or gastrointestinal infections [23]. Thus, IgAN has traditionally been considered as correlated to mucosal infections. Mucosal immunity is triggered by recognition of bacterial or viral nucleic acids or products or other pathogen-associated molecular patterns (PAMPs) by toll-like receptors (TLRs) [24]. The TLR activation triggers a cascade of intracellular messages leading to activation of the interferon regulatory factor (IRF) and the NF-KB. The Stat-1/ IRF-1 dimers are the major transcription factors involved in IFN- $\gamma$ signal transduction. Under the stimuli of IFN- $\gamma$, the expression level of 
immunoproteasome subunits was significantly upregulated. This cytokine-induced expression is the result of binding Stat-1 and IRF-1 transcription factors to multiple IFN- $\gamma$ consensus/activation sequences in the promoter region of the $L M P 2, L M P 7$, and MECL-1 genes [25]. In agreement, it has been reported that genes involved in the immunoproteasome pathway were activated in IgAN during phases of clinical activity [10]. Coppo, et al. [10] observed upregulated expression of the immunoproteasome subunits mRNA in PBMC from patients with IgAN. However, precise molecular mechanism of the upregulation of immunoproteasome and how it is involved in pathogenesis and/or progression of IgAN remain obscure.

Recent studies show both pathogenic and prognostic role of anti-glycan antibodies in IgAN, suggesting that $\operatorname{IgAN}$ is a type of autoimmune disease [26-28]. Aberrantly glycosylated IgA1 might be recognized as an autoantigen, and thus the immune response against it may lead to the production of anti-IgA1-antibodies. However, of interest, more recent studies suggest that some pathogens such as Epstein-Barr virus and Streptococcus bacteria possess GalNAc-containing structures on their surfaces, which might subsequently cross-react with glycans on IgA1 resulting in the formation of pathological immune complexes [29-31]. This 'molecular mimicry' might explain the association of macroscopic hematuria with upper respiratory tract infections [23]. During intracellular viral or bacterial infection period, it is supposed that enhanced immunoproteasome activation in IgAN may lead to an increased presentation of self-antigens onto MHC-I molecules, ultimately resulting in enhanced autoimmune responses. In this process, the immunoproteasome may cleave either endogenous or exogenous proteins to generate antigen peptides, which are firstly complexed to MHC-I in the endoplasmic reticulum and then exposed on the plasma membrane of antigen-presenting cells (APCs), for either direct or cross-presentation to CD8+ T-lymphocytes[21]. Owing to unique structural features and proteolytic activity of the immunoproteasome, the process is accomplished at a higher rate and with greater efficacy compared with constitutive proteasome.

\section{Cytokine production facilitation}

As mentioned above, the TLR activation triggers a cascade of intracellular messages leading to

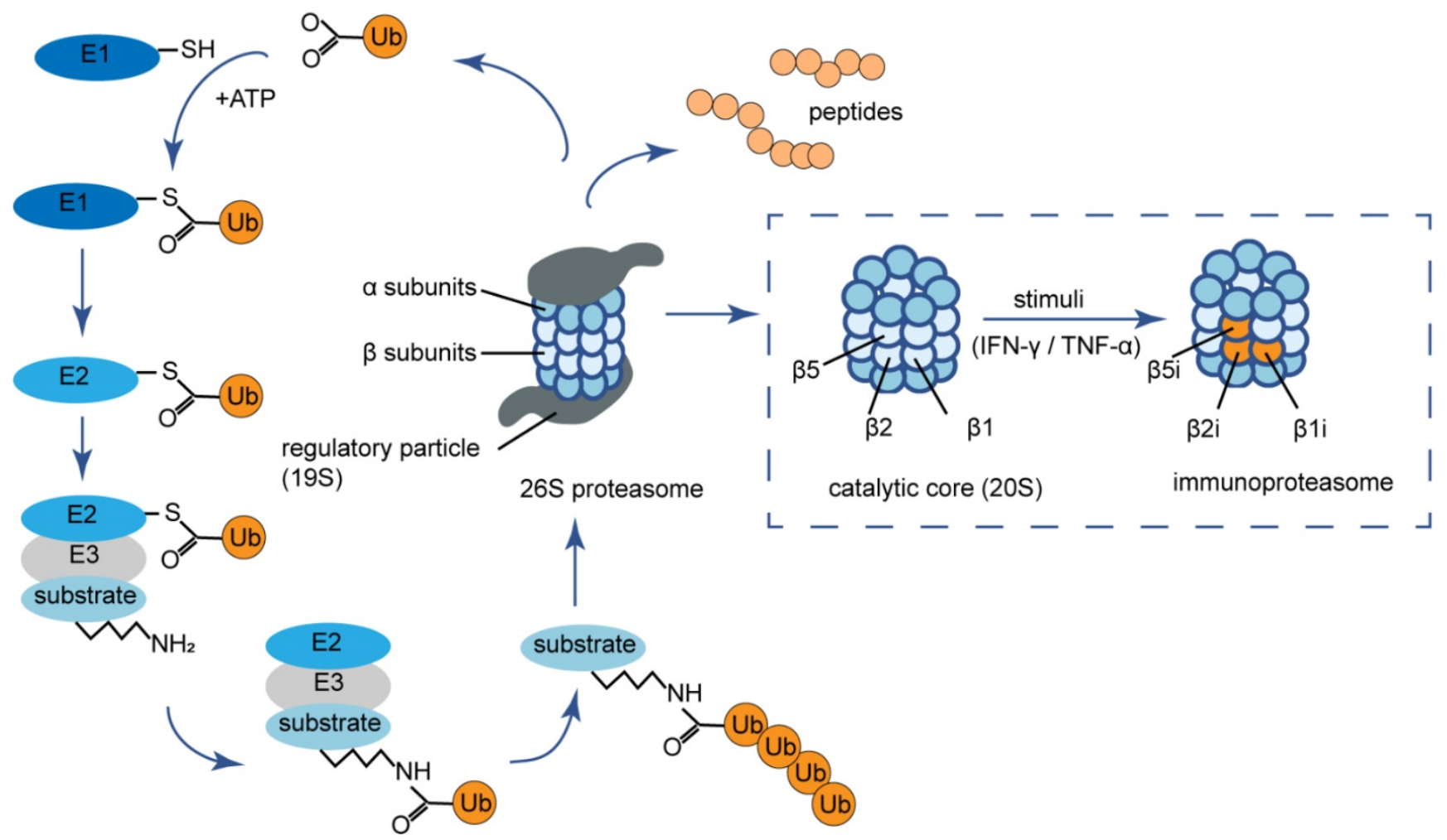

Figure 1. The ubiquitin-proteasome system and (immuno) proteasome composition. The ubiquitin-proteasome system is an ubiquitin-dependent proteolysis system. Substrates are first covalently attached to multiple ubiquitin moieties via the action of ubiquitin-activating enzymes (E1), ubiquitin-conjugating enzymes (E2) and ubiquitin ligases (E3) in an ATP-dependent manner. Poly-ubiquitinated proteins are then degraded by the $26 \mathrm{~S}$ proteasome, which consists of a catalytic core complex, that is, the multi-subunit $20 \mathrm{~S}$ proteasome (either constitutive or immunoproteasome) associated with two $19 \mathrm{~S}$ regulator complexes governing the access to its catalytic cavity. The barrel shaped constitutive $20 \mathrm{~S}$ proteasome is formed by four stacked rings of seven subunits each with the arrangement of $\alpha 1-\alpha 7$ or $\beta 1-\beta 7$. Under the stimuli of certain pro-inflammatory cytokines such as IFN- $\gamma$ and TNF- $\alpha$, the $\beta 1, \beta 2$, and $\beta 5$ subunits are replaced by $\beta 1 i$ (LMP2), $\beta 2 i$ (MECL-1) and $\beta 5 i$ (LMP7) respectively, forming the variant immunoproteasome. Ub: ubiquitin; ATP: adenosine triphosphate; IFN-ү: interferon-ү; TNF- $\alpha$ : tumor necrosis factor- $\alpha$. 
activation of the NF-kB transcription pathway [24]. Coppo, et al. [10] noticed that nuclear translocation of NF-kB p50 and p65 were significantly enhanced in patients with IgAN compared to healthy controls. As known, activation of NF-kB leads to release of cytokines and chemokines and killing pathogens via the oxidative burst [24]. Also, cytokines might participate in an aberrant IgA1 galactosylation. For example, IL-17 downregulates the expression of C1GalT1 and C1GalT1 specific chaperone 1 (C1GalT1C1), leading to higher secretion of Gd-IgA1 from B cells [32]. And IL-6, promotes hypogalactosylation of IgA1 by increasing ST6GalNAc-II activity and decreasing activity of C1GalT1 [33] (Fig. 2). Recent studies have elucidated roles of the immunoproteasome beyond antigen presentation. The enhanced immunoproteasome activation in IgAN may be associated with regulating inflammatory pathways, through a direct effect on NF-kB signaling and the production of proinflammatory cytokines [25, 34, 35].

NF-kB is a pivotal transcription factor that promotes growth factors, proinflammatory cytokines, and cell survival signaling. Evidence from immunoproteasome-deficient mice has provided compelling support for the role of immunoproteasome in NF-KB activation. It was observed that cell lines lacking LMP2 had defects in processing NF-kB precursors ( $100 / \mathrm{p} 105)$ to the active transcription factors (p52/p50) [25]. Furthermore, in preclinical animal models, selective immunoproteasome inhibitors were found to reduce the secretion of proinflammatory TNF-a, IL-6, and IL-23 through altering NF-KB activity [22]. Several mutations in the human PSMB8 and PSMB9 genes encoding the LMP7 and LMP2 immunoproteasome subunits, respectively, have been discovered in autoinflammatory disorders $[17,36]$. In cells from patients with PSMB8-associated mutations in LMP7, basal IL-6 production was significantly higher compared to healthy controls [36]. These examples show that the immunoproteasome content can affect cytokine production. And compared with the constitutive proteasome (nearly 82 minutes), the decreased time in which immunoproteasome can be formed (only 21 minutes) makes the immunoproteasome provide a rapid response to inflammation or stress as signaled by proinflammatory cytokines [22].
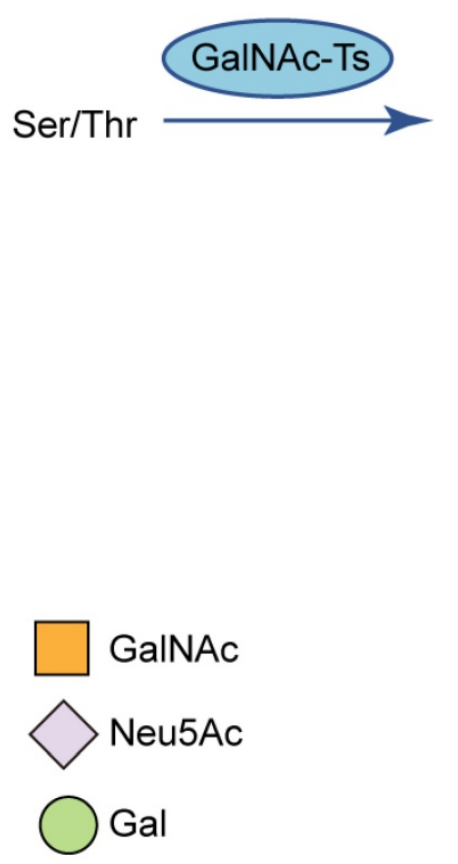
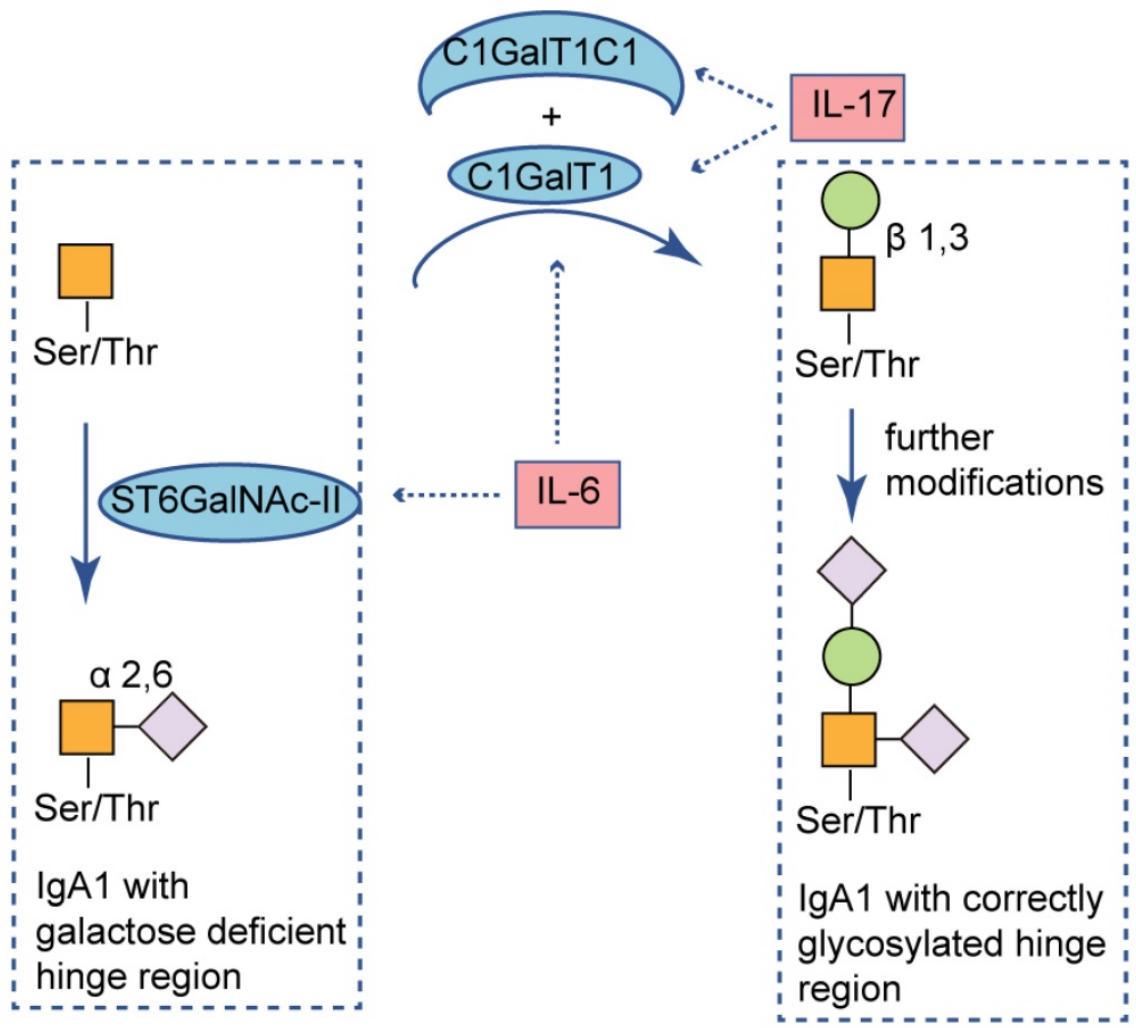

Figure 2. Immunoproteasome-related cytokines alter IgAl O-glycosylation. O-glycans are synthesized in a stepwise manner, starting with attachment of $\mathrm{N}$-acetylgalactosamine to Ser/Thr residues of the hinge region (catalyzed by GalNAc-Ts), followed by addition of galactose (catalyzed by C1GalT1 and its chaperone C1GalT1C1). Sialic acid can be added to each glycan by ST6GalNAC-II for attachment to GaINAc and this can prevent subsequent addition of galactose. IL-17 decreases expression of $\mathrm{ClGaIT1}$ and its chaperon $\mathrm{ClGALT1Cl}$; IL-6 increases expression of ST6GaINAC-II and decreases expression of C1GalT1. Both of the immunoproteasome-related cytokines can stimulate production of Gd-lgA1. Ser: serine; Thr: threonine; GalNAc: N-acetylgalactosaminyl; Neu5Ac: N-acetylneuraminic acid; Gal: galactose; GaINAc-Ts: N-acetylgalactosaminyl-transferases; ST6GaINAc-II: $\alpha$ - $N$-acetylgalactosaminide $\alpha-2,6$-sialyltransferase 2; C1GalT1: core 1 $\beta 1,3$-galactosyltransferase; CIGaIT1C1: C1GalT1 chaperone 1; IL-17: interleukin-17; IL-6: interleukin-6. 


\section{$T$ cell differentiation}

As an immunological disorder, accumulating research evidence supports the involvement of multiple abnormal lymphocyte subsets in IgAN. Although a heterogeneous disease, a report observed higher proportions of circulatory Th2 and Th17 cells, lower Th1 and Treg cells in patients with IgAN [31]. Recent studies show that immunoproteasome activity promotes helper $\mathrm{T}(\mathrm{Th})$ cell differentiation (including proinflammatory Th1 and Th17 cells) and effector T cell expansion (cytotoxic CD8 cells), while repressing regulatory $\mathrm{T}$ (Treg) cell induction [22]. And the immunoproteasome subunit LMP7, coded by PSMB8 gene, is necessary for Th17 differentiation and inhibits Treg differentiation [37]. It was found that PBMC of IgAN patients had higher expression of PSMB8 gene, especially those individuals with high proteinuria [9]. And GWAS reported protective genotypes in IgAN showed lower PSMB8 gene expression [3]. Thus, the immunoproteasome has been proposed to perform specialized roles in $\mathrm{T}$ cell differentiation and it may participate in pathogenesis and pathophysiology of IgAN [37]. Enhanced immunoproteasome activation may contribute to Th17/Treg disequilibrium in IgAN. Apart from participation in the immune response against infection, cytokines like IL-17 can also impact aberrant IgA1 galactosylation in IgAN [33]. In IgAN, higher proportions of Th17 lymphocytes secrete high concentrations of IL-17, which can stimulate production of Gd-IgA1. However, Treg cells cannot effectively suppress the defective immune response leading to formation of immune complexes. All these processes can cause formation and deposition of circulating immune complexes in glomeruli [31]. In agreement, Lin, et al. [38] demonstrated that IgAN patients had an imbalance of Treg/Th17 and in particular, the altered distribution of Treg and Th17 in circulation and local renal tissues were correlated with prognostic parameters of IgAN such as impaired glomerular filtration rate (GFR), proteinuria, high blood pressure and tubulointerstitial injury.

\section{B cell immunity}

In IgAN, some data suggest bacterial and viral antigens from antigens involved in upper respiratory and gastrointestinal mucosal infections could be found in the glomeruli in association with $\operatorname{IgA}$ deposits [23]. After encountering an antigen, Blymphocytes activate a complex program involving proliferation, generation of memory cells, isotype switch, and affinity maturation [39]. Ultimately, B cells differentiate into plasma cells (PC) and produce antibodies to control and eliminate infection. The increased serum levels of Gd-IgA1, glycan-specific
$\operatorname{Ig} \mathrm{A}$ and $\operatorname{IgG}$ autoantibodies in $\operatorname{Ig} \mathrm{AN}$ patients were produced by plasma cells (Fig. 3). Although some of them home in the bone marrow and survive for longer periods, most plasma cells are short-lived after a few days of intense immunoglobulin (Ig) secretion [39]. Research has demonstrated that B cells require complex regulation of proteasome activity during differentiation and secretion of antibodies [40]. The limiting plasma cell lifespan may be caused by a dramatic decrease of the relative amount and proteolytic activity of proteasome in the late phases of plasmacytic differentiation.

In general, the proteasome can degrade endoplasmic reticulum (ER) proteins which failed to fold properly [41], and this termed as ER-associated degradation (ERAD). Moreover, the proteasome plays an important role in unfolded protein response (UPR). The signaling pathway called UPR ensures that plasma cells can handle the proper folding of Ig proteins. When the ER transmembrane endoribonuclease and kinase called IRE1 senses unfolded proteins, it oligomerizes and is activated by autophosphorylation, thus allowing it to excise an intron of the long isoform of X-box binding protein 1 (XBP-1 $\mu)$, creating a short isoform of XBP-1 (XBP-1s) [42]. The XBP-1 $\mu$ protein, which could be degraded through the ubiquitin-proteasome pathway, can act as a dominant-negative inhibitor of XBP-1s activity [43]. In addition to quieting ER stress by activating the UPR, XBP-1s is also a transcription factor known to be selectively and specifically required for the terminal differentiation of B lymphocytes to plasma cells [44]. However, it was surprising to observe that the enzymatic activity and the relative abundance of proteasome decreased during plasma cell differentiation [39]. With progressive fall of proteolytic capacity, accumulated ERAD substrates and $\mathrm{XBP}-1 \mu$ protein and suppressed $\mathrm{XBP}-1$ s activity together lead to ER stress and cell apoptosis. Thus, the apoptosis of plasma cell was promoted by an unbalance between load (immunoglobulin synthesis) and capacity of the proteolytic machinery (proteasome).

It is speculated that the upregulation of the immunoproteasome in IgAN may partly balance the unfavorable proteasomal load/capacity ratio and prolong plasma cells survival. Similar to the constitutive proteasome, the immunoproteasome can also degrade ERAD substrates, misfolded and or damaged proteins to limit the accumulation of unwanted proteins and decrease cell apoptosis [45]. On the other hand, it has been reported that immunoproteasome-deficient mice have altered NF-KB activity, leading to compromised B cell numbers, survival, and function [34]. Apart from this, 
there is increasing recognition for the presence of a mucosa-bone marrow axis in the pathogenesis of IgAN [46]. Large numbers of polymeric IgA1-positive plasma cells are found in the bone marrow (BM) in IgAN and these long-lived plasma cells may maintain a high level of IgA1 in the blood [47]. It is clear that extrinsic factors such as cytokines play important roles in shaping the environmental niche that promotes survival of PC in the BM. Intriguingly, at least two key cytokines contributing to the plasma cell niche, TNF and IL-6, have been demonstrated to be dependent upon the immunoproteasome subunit LMP7 [48]. In agreement with this notion, Li, et al. [49] reported that immunoproteasome inhibitor ONX 0914 can induce plasma cell apoptosis through activating the UPR and suppressing plasma cell survival factors in the bone marrow.

\section{The immunoproteasome as a therapeutic target}

In recent years, studies have elucidated roles of the immunoproteasome beyond antigen presentation. The immunoproteasome participates in a variety of biological processes, including protein quality control, transcription, immune response, cell signaling, and apoptosis and so on [50]. The immunoproteasome dysfunction or dysregulation, therefore, is closely related to a wide range of diseases such as tumor,

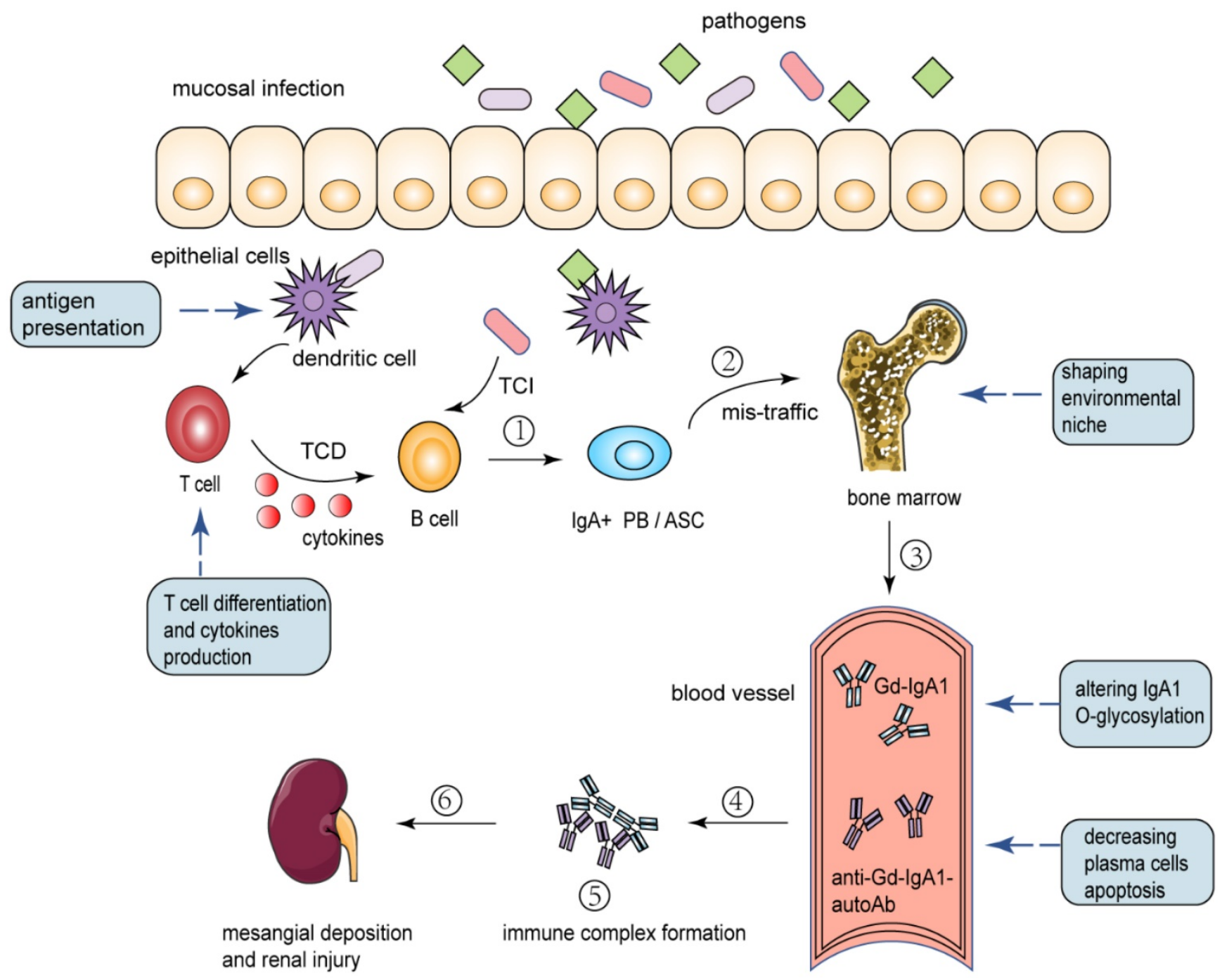

Figure 3. Proposed pathways the immunoproteasome involved in the pathogenesis of IgAN. (1) Mucosal infection primes naive $B$ cells to class switch to become $\lg \mathrm{A}^{+}$plasmablasts (PB) and antibody-secreting cells (ASC) through T-cell-dependent (TCD) and T-cell-independent (TCl) processes. Antigen presentation by immunoproteasome promotes immune response, T cell differentiation and cytokines production. (2) A proportion of antigen-committed IgA PB and ASC with altered homing receptors and therefore they mistakenly "home" to the bone marrow. Immunoproteasome-related cytokines can contribute to the environmental niche in bone marrow. (3) These translocated cells take up residence in the bone marrow, where they secrete IgA into the systemic circulation. Factors such as genetic background and immunoproteasome-related cytokines can alter IgAl O-glycosylation and lead to Gd-lgAl production. (4) Plasma cells secrete IgG and IgA autoantibodies directed against the Gd-lgAl hinge region O-glycans. In the phase of intense secretion of antibodies, immunoproteasome can balance the unfavorable proteasomal load/capacity ratio and decrease plasma cells apoptosis. (5) Formation of immune complexes from autoantigen (Gd-lgAl) and O-glycan-specific antibodies. (6) Deposition of pathogenic immune complexes in the mesangium, activation of mesangial cells, and induction of glomerular injury. PB: plasmablasts; ASC: antibody-secreting cells; TCD: T-cell-dependent; TCl: T-cell-independent; Gd-lgA1: galactose-deficient IgA1; Anti-Gd-lgA1-autoAb: Anti-Gd-lgA1-autoantibody. 
infectious diseases, central nervous system diseases, autoimmune and inflammatory diseases [16]. As a result, significant research has focused on elucidating the role of the immunoproteasome in a variety of diseases to evaluate its potential as a therapeutic target. Indeed, the proteasome inhibitor bortezomib has long been on the market as an Food and Drug Administration (FDA)-approved therapy for multiple myelomas, and many other proteasome inhibitors are being investigated [51]. However, most of these inhibitors alter the catalytic activity of both the constitutive proteasome and the immunoproteasome indiscriminately, leading to various unwanted side effects and the application has been limited [52]. To overcome these off-target effects, efforts are being made to design selective inhibitors that specifically target immunoproteasome subunits. The immunoproteasome-specific therapy can selectively affect the function of activated immune cells while sparing other cell types that would be damaged by bortezomib [53]. In preclinical animal models, selective immunoproteasome inhibitors are beneficial to the treatment of many different diseases including hematologic malignancies, experimental colitis, experimental arthritis, systemic lupus erythematosus, and multiple sclerosis [48, 54-57]. These may be because the immunoproteasome inhibitors affected a multitude of cellular responses such as endoplasmatic reticulum stress, unfolded protein response, NF-KB inhibition, cytokine modulation, $\mathrm{T}$ cell differentiation, or an increase in proapoptotic factors and tumor suppressors [58]. In addition to the diseases in which the level of the immunoproteasome expression or activity is higher than normal, there are also different diseases in which the immunoproteasome is hindered, which may benefit from the stimulation of immunoproteasome activity. One possible class of diseases that could benefit from immunoproteasome stimulation is viral infections [16].

Notably, several interesting trials testing the effect of proteasome inhibitor in patients with IgAN and significant proteinuria have been performed [15, 59]. The outcomes of a single-center open-label pilot trial showed the potential of therapy targeted at (immuno) proteasome in IgAN treatment. After 1-year follow-up, of all 8 subjects who received and tolerated 4 doses of bortezomib over a 2-week period during enrollment, 3 subjects (38\%) had complete remission defined as proteinuria of less than $300 \mathrm{mg}$ per day [15]. However, the trial has several significant limitations, including small sample size, no control group and nonrandomization. Besides, bortezomib targets proteasome subunits $\beta 5, \beta 5 i$ and $\beta 1 i$, and alters the catalytic activity of constitutive proteasome and immunoproteasome indiscriminately. Thus, more evidence is needed to verify the conclusion of this trial and the efficacy of (immuno) proteasome inhibition in $\operatorname{IgAN}$. As an autoimmune disease elicited by Gd-IgA1 antibodies, immunoproteasome inhibitors may be considered as a promising strategy due to its important role in plasma cells survival [49]. However, before the immunoproteasome becomes a therapeutic target in IgAN, further research is necessary to clarify whether and how the immunoproteasome participate in pathogenesis and/or progression of the disease. The advantages and disadvantages of immunoproteasome as a therapeutic target are summarized in Table 1 [60-67].

Table 1. Advantages and disadvantages of immunoproteasome as a therapeutic target

\begin{tabular}{|c|c|}
\hline Advantages & Descriptions \\
\hline Less off-target effects & $\begin{array}{l}\text { The immunoproteasome-specific therapy } \\
\text { targets subunits of immunoproteasome }\end{array}$ \\
\hline $\begin{array}{l}\text { Reducing the toxic and side } \\
\text { effects }\end{array}$ & $\begin{array}{l}\text { The immunoproteasome-specific therapy } \\
\text { selectively affects the function of activated } \\
\text { immune cells while sparing other cell types }\end{array}$ \\
\hline Efficient immune regulation & $\begin{array}{l}\text { The immunoproteasome participates in a } \\
\text { variety of immune processes }\end{array}$ \\
\hline $\begin{array}{l}\text { Broad biological } \\
\text { applications }\end{array}$ & $\begin{array}{l}\text { Recent studies confirm that the } \\
\text { immunoproteasome is related to many human } \\
\text { diseases such as autoimmune diseases, central } \\
\text { nervous system diseases and hematologic } \\
\text { malignancies[60-63] }\end{array}$ \\
\hline Less drug resistance & $\begin{array}{l}\text { The potential of selective immunoproteasome } \\
\text { inhibitors in overcoming drug resistance has } \\
\text { been reported }[64,65]\end{array}$ \\
\hline Disadvantages & Descriptions \\
\hline $\begin{array}{l}\text { Further basic research is } \\
\text { needed }\end{array}$ & $\begin{array}{l}\text { More basic studies on immunoproteasome } \\
\text { structure and functions are needed to guide } \\
\text { immunoproteasome-specific therapy }[66,67]\end{array}$ \\
\hline $\begin{array}{l}\text { Influence on some } \\
\text { physiological processes }\end{array}$ & $\begin{array}{l}\text { Apart from immune response, } \\
\text { immunoproteasome is involved in some } \\
\text { physiological processes such as protein } \\
\text { degradation, cell proliferation and survival }\end{array}$ \\
\hline
\end{tabular}

\section{Future directions}

In this review, we discuss the important role of immunoproteasome in several mechanisms respectively and it is likely that these mechanisms synergistically contribute to the susceptibility to IgAN and risk of disease progression. The dysfunction or dysregulation of the immunoproteasome may play a crucial role in IgAN pathogenesis and/or progression (Fig. 3). However, clinical implications of the immunoproteasome in IgAN are still largely unexplored. There is much to be done in this area. For instance, areas of interest might include the following: (i) genetic analysis of the variation of immunoproteasome components and their correlation with immunoproteasome function; (ii) determining whether and how immunoproteasome impact the pathogenesis of IgAN; (iii) development of IgAN animal models that harbor mutations in the immuno- 
proteasome gene; (iv) determining whether immunoproteasome can serve as a biomarker for clinical activity and aid in genetic screening of IgAN susceptible groups; (v) studying the potential of immunoproteasome as a therapeutic target in IgAN. Elucidating the exact pathological contribution of immunoproteasome dysfunction or dysregulation to the development of IgAN and the underlying causal mechanisms will undoubtedly bring valuable insights into the pathogenesis of IgAN and lead to the development of novel therapeutic approaches.

\section{Abbreviations}

IgAN: IgA nephropathy; MHC-I: major histocompatibility complex-I; CKD: chronic kidney disease; ESKD: end stage kidney disease; Gd-IgA1: galactose-deficient IgA1; GWAS: genome-wide association studies; SNP: single nucleotide polymorphisms; LMP: low molecular mass polypeptide; PBMC: peripheral blood mononuclear cells; SLE: systemic lupus erythematosus; NF-KB: nuclear factor kappa B; UPS: ubiquitin-proteasome system; IFN: interferon; TNF: tumor necrosis factor; MECL-1: multicatalytic endopeptidase complexlike-1; PAMPs: pathogen-associated molecular patterns; TLRs: toll-like receptors; IRF: interferon regulatory factor; APCs: antigen-presenting cells; IL-17: interleukin-17; C1GalT1C1: C1GalT1 specific chaperone 1; Th cell: helper $\mathrm{T}$ cell; Treg cell: regulatory $\mathrm{T}$ cell; GFR: glomerular filtration rate; PC: plasma cells; Ig: immunoglobulin; ER: endoplasmic reticulum; ERAD: endoplasmic reticulum associated degradation; UPR: unfolded protein response; XBP-1: X-box binding protein 1; BM: bone marrow; FDA: Food and Drug Administration.

\section{Acknowledgements}

Support was provided by Beijing Natural Science Foundation (Z190023); National Science Foundation of China (Grant 81970613); Beijing Nova Program Interdisciplinary Cooperation Project (Z191100001119004); Beijing Youth Top-notch Talent Support Program (2017000021223ZK31); Clinical Medicine Plus X-Young Scholars Project of Peking University (PKU2020LCXQ003); the University of Michigan Health System-Peking University Health Science Center Joint Institute for Translational and Clinical Research (BMU2017JI007); Chinese Academy of Medical Sciences Research Unit (2019RU023); CAMS Innovation Fund for Medical Sciences (2019-I2M-5-046). The funders had no role in study design, data collection and analysis, decision to publish, or preparation of the manuscript.

\section{Competing Interests}

The authors have declared that no competing interest exists.

\section{References}

1. Rodrigues JC, Haas M, Reich HN. IgA Nephropathy. Clinical Journal of the American Society of Nephrology. 2017; 12: 677.

2. Lai KN, Tang SCW, Schena FP, Novak J, Tomino Y, Fogo AB, et al. IgA nephropathy. Nature Reviews Disease Primers. 2016; 2: 16001

3. Suzuki H, Kiryluk K, Novak J, Moldoveanu Z, Herr AB, Renfrow MB, et al. The pathophysiology of IgA nephropathy. J Am Soc Nephrol. 2011; 22: 1795-803.

4. Gharavi AG, Kiryluk K, Choi M, Li Y, Hou P, Xie J, et al. Genome-wide association study identifies susceptibility loci for IgA nephropathy. Nat Genet. 2011; 43: 321-7.

5. Yu XQ, Li M, Zhang H, Low HQ, Wei X, Wang JQ, et al. A genome-wide association study in Han Chinese identifies multiple susceptibility loci for IgA nephropathy. Nat Genet. 2011; 44: 178-82.

6. Li M, Foo JN, Wang JQ, Low HQ, Tang XQ, Toh KY, et al. Identification of new susceptibility loci for IgA nephropathy in Han Chinese. Nat Commun. 2015; 6: 7270 .

7. Kiryluk K, Li Y, Scolari F, Sanna-Cherchi S, Choi M, Verbitsky M, et al. Discovery of new risk loci for IgA nephropathy implicates genes involved in immunity against intestinal pathogens. Nat Genet. 2014; 46: 1187-96.

8. Feehally J, Farrall M, Boland A, Gale DP, Gut I, Heath S, et al. HLA has strongest association with IgA nephropathy in genome-wide analysis. J Am Soc Nephrol. 2010; 21: 1791-7.

9. Tang SC, Lai KN. The ubiquitin-proteasome pathway and IgA nephropathy: a novel link? Kidney Int. 2009; 75: 457-9.

10. Coppo R, Camilla R, Alfarano A, Balegno S, Mancuso D, Peruzzi L, et al. Upregulation of the immunoproteasome in peripheral blood mononuclear cells of patients with IgA nephropathy. Kidney Int. 2009; 75: 536-41.

11. Peruzzi L, Coppo R, Cocchi E, Loiacono E, Bergallo M, Bodria M, et al. The switch from proteasome to immunoproteasome is increased in circulating cells of patients with fast progressive immunoglobulin A nephropathy and associated with defective CD46 expression. Nephrol Dial Transplant. 2020.

12. Zhou XJ, Cheng FJ, Zhu L, Lv JC, Qi YY, Hou P, et al. Association of systemic lupus erythematosus susceptibility genes with IgA nephropathy in a Chinese cohort. Clin J Am Soc Nephrol. 2014; 9: 788-97.

13. Lewis MJ, Vyse S, Shields AM, Boeltz S, Gordon PA, Spector TD, et al. UBE2L3 polymorphism amplifies NF-kB activation and promotes plasma cell development, linking linear ubiquitination to multiple autoimmune diseases. Am J Hum Genet. 2015; 96: 221-34.

14. Lafayette RA, Canetta PA, Rovin BH, Appel GB, Novak J, Nath KA, et al. A Randomized, Controlled Trial of Rituximab in IgA Nephropathy with Proteinuria and Renal Dysfunction. J Am Soc Nephrol. 2017; 28: 1306-13.

15. Hartono C, Chung M, Perlman AS, Chevalier JM, Serur D, Seshan SV, et al. Bortezomib for Reduction of Proteinuria in IgA Nephropathy. Kidney Int Rep. 2018; 3: 861-6.

16. Zerfas BL, Maresh ME, Trader DJ. The Immunoproteasome: An Emerging Target in Cancer and Autoimmune and Neurological Disorders. J Med Chem. 2020; 63: 1841-58.

17. Yasutomo K. Dysregulation of immunoproteasomes in autoinflammatory syndromes. Int Immunol. 2019; 31: 631-7.

18. Xi J, Zhuang R, Kong L, He R, Zhu H, Zhang J. Immunoproteasome-selective inhibitors: An overview of recent developments as potential drugs for hematologic malignancies and autoimmune diseases. Eur J Med Chem. 2019; 182: 111646 .

19. Yao L, Zhou L, Xuan Y, Zhang P, Wang X, Wang T, et al. The proteasome activator REGgamma counteracts immunoproteasome expression and autoimmunity. J Autoimmun. 2019; 103: 102282.

20. Zhang $\mathrm{C}$, Zhu $\mathrm{H}$, Shao J, He R, Xi J, Zhuang $\mathrm{R}$, et al. Immunoproteasome-selective inhibitors: the future of autoimmune diseases? Future Med Chem. 2020; 12: 269-72.

21. Limanaqi F, Biagioni F, Gaglione A, Busceti CL, Fornai F. A Sentinel in the Crosstalk Between the Nervous and Immune System: The (Immuno)-Proteasome. Front Immunol. 2019; 10: 628.

22. Eskandari SK, Seelen MAJ, Lin G, Azzi JR. The immunoproteasome: An old player with a novel and emerging role in alloimmunity. Am J Transplant. 2017; 17: 3033-9.

23. Rollino C, Vischini G, Coppo R. IgA nephropathy and infections. Journal of Nephrology. 2016; 29: 463-8.

24. Vergano L, Loiacono E, Albera R, Coppo R, Camilla R, Peruzzi L, et al. Can tonsillectomy modify the innate and adaptive immunity pathways involved in IgA nephropathy? Journal of Nephrology. 2015; 28: 51-8.

25. Ferrington DA, Gregerson DS. Immunoproteasomes: structure, function, and antigen presentation. Prog Mol Biol Transl Sci. 2012; 109: 75-112

26. Kokubo T, Hashizume K, Iwase H, Arai K, Tanaka A, Toma K, et al. Humoral immunity against the proline-rich peptide epitope of the IgA1 hinge region in IgA nephropathy. Nephrol Dial Transplant. 2000; 15: 28-33. 
27. Suzuki H, Fan R, Zhang Z, Brown R, Hall S, Julian BA, et al. Aberrantly glycosylated $\operatorname{IgA} 1$ in IgA nephropathy patients is recognized by IgG antibodies with restricted heterogeneity. J Clin Invest. 2009; 119: 1668-77.

28. Tomana M, Novak J, Julian BA, Matousovic K, Konecny K, Mestecky J. Circulating immune complexes in IgA nephropathy consist of IgA1 with galactose-deficient hinge region and antiglycan antibodies. J Clin Invest. 1999; 104: 73-81.

29. Mestecky J, Novak J, Moldoveanu Z, Raska M. IgA nephropathy enigma. Clin Immunol. 2016; 172: 72-7.

30. Novak J, Julian BA, Tomana M, Mestecky J. IgA glycosylation and IgA immune complexes in the pathogenesis of IgA nephropathy. Semin Nephrol. 2008; $28: 78-87$.

31. Ruszkowski J, Lisowska KA, Pindel M, Heleniak Z, Debska-Slizien A, Witkowski JM. T cells in IgA nephropathy: role in pathogenesis, clinical significance and potential therapeutic target. Clin Exp Nephrol. 2019; 23: 291-303.

32. Lin JR, Wen J, Zhang H, Wang L, Gou FF, Yang M, et al. Interleukin-17 promotes the production of underglycosylated IgA1 in DAKIKI cells. Ren Fail. 2018; 40: 60-7.

33. Suzuki H, Raska M, Yamada K, Moldoveanu Z, Julian BA, Wyatt RJ, et al. Cytokines alter IgA1 O-glycosylation by dysregulating C1GalT1 and ST6GalNAc-II enzymes. J Biol Chem. 2014; 289: 5330-9.

34. Hensley SE, Zanker D, Dolan BP, David A, Hickman HD, Embry AC, et al. Unexpected role for the immunoproteasome subunit LMP2 in antiviral humoral and innate immune responses. J Immunol. 2010; 184: 4115-22.

35. Kaur G, Batra S. Emerging role of immunoproteasomes in pathophysiology. Immunol Cell Biol. 2016; 94: 812-20.

36. Kitamura A, Maekawa Y, Uehara H, Izumi K, Kawachi I, Nishizawa M, et al. A mutation in the immunoproteasome subunit PSMB8 causes autoinflammation and lipodystrophy in humans. J Clin Invest. 2011; 121: 4150-60.

37. Kalim KW, Basler M, Kirk CJ, Groettrup M. Immunoproteasome subunit LMP7 deficiency and inhibition suppresses Th1 and Th17 but enhances regulatory T cell differentiation. J Immunol. 2012; 189: 4182-93.

38. Lin FJ, Jiang GR, Shan JP, Zhu C, Zou J, Wu XR. Imbalance of regulatory T cells to Th17 cells in IgA nephropathy. Scand J Clin Lab Invest. 2012; 72: 221-9.

39. Cenci S, Mezghrani A, Cascio P, Bianchi G, Cerruti F, Fra A, et al. Progressively impaired proteasomal capacity during terminal plasma cell differentiation. Embo j. 2006; 25: 1104-13.

40. Cascio P, Oliva L, Cerruti F, Mariani E, Pasqualetto E, Cenci S, et al. Dampening $\mathrm{Ab}$ responses using proteasome inhibitors following in vivo $\mathrm{B}$ cell activation. Eur J Immunol. 2008; 38: 658-67.

41. Fregno I, Molinari M. Proteasomal and lysosomal clearance of faulty secretory proteins: ER-associated degradation (ERAD) and ER-to-lysosome-associated degradation (ERLAD) pathways. Crit Rev Biochem Mol Biol. 2019; 54: 153-63.

42. Gommerman JL, Rojas OL, Fritz JH. Re-thinking the functions of $\operatorname{IgA}(+)$ plasma cells. Gut Microbes. 2014; 5: 652-62.

43. Lee AH, Iwakoshi NN, Anderson KC, Glimcher LH. Proteasome inhibitors disrupt the unfolded protein response in myeloma cells. Proc Natl Acad Sci U A. 2003; 100: 9946-51.

44. Reimold AM, Iwakoshi NN, Manis J, Vallabhajosyula P, Szomolanyi-Tsuda E, Gravallese EM, et al. Plasma cell differentiation requires the transcription factor XBP-1. Nature. 2001; 412: 300-7.

45. Nathan JA, Spinnenhirn V, Schmidtke G, Basler M, Groettrup M, Goldberg AL. Immuno- and constitutive proteasomes do not differ in their abilities to degrade ubiquitinated proteins. Cell. 2013; 152: 1184-94.

46. Suzuki Y, Tomino Y. Potential immunopathogenic role of the mucosa-bone marrow axis in IgA nephropathy: insights from animal models. Semin Nephrol. 2008; 28: 66-77.

47. van den Wall Bake AW, Daha MR, Evers-Schouten J, van Es LA. Serum IgA and the production of IgA by peripheral blood and bone marrow lymphocytes in patients with primary IgA nephropathy: evidence for the bone marrow as the source of mesangial IgA. Am J Kidney Dis. 1988; 12: 410-4.

48. Ichikawa HT, Conley T, Muchamuel T, Jiang J, Lee S, Owen T, et al. Beneficial effect of novel proteasome inhibitors in murine lupus via dual inhibition of type I interferon and autoantibody-secreting cells. Arthritis Rheum. 2012; 64: 493-503.

49. Li J, Koerner J, Basler M, Brunner T, Kirk CJ, Groettrup M. Immunoproteasome inhibition induces plasma cell apoptosis and preserves kidney allografts by activating the unfolded protein response and suppressing plasma cell survival factors. Kidney Int. 2019; 95: 611-23.

50. Angeles A, Fung G, Luo H. Immune and non-immune functions of the immunoproteasome. Front Biosci (Landmark Ed). 2012; 17: 1904-16.

51. Blade J, Cibeira MT, Rosinol L. Bortezomib: a valuable new antineoplastic strategy in multiple myeloma. Acta Oncol. 2005; 44: 440-8.

52. Cengiz Seval G, Beksac M. The safety of bortezomib for the treatment of multiple myeloma. Expert Opin Drug Saf. 2018; 17: 953-62.

53. Murata S, Takahama Y, Kasahara M, Tanaka K. The immunoproteasome and thymoproteasome: functions, evolution and human disease. Nat Immunol. 2018; 19: 923-31.

54. Basler M, Dajee M, Moll C, Groettrup M, Kirk CJ. Prevention of experimental colitis by a selective inhibitor of the immunoproteasome. J Immunol. 2010; 185: 634-41.

55. Bellavista E, Santoro A, Galimberti D, Comi C, Luciani F, Mishto M. Current understanding on the role of standard and immunoproteasomes in inflammatory/immunological pathways of multiple sclerosis. Autoimmune Dis. 2014; 2014: 739705

56. Ettari R, Zappala M, Grasso S, Musolino C, Innao V, Allegra A. Immunoproteasome-selective and non-selective inhibitors: A promising approach for the treatment of multiple myeloma. Pharmacol Ther. 2018; 182: $176-92$.

57. Muchamuel T, Basler M, Aujay MA, Suzuki E, Kalim KW, Lauer C, et al. A selective inhibitor of the immunoproteasome subunit LMP7 blocks cytokine production and attenuates progression of experimental arthritis. Nat Med. 2009; 15: 781-7.

58. Cromm PM, Crews CM. The Proteasome in Modern Drug Discovery: Second Life of a Highly Valuable Drug Target. ACS Cent Sci. 2017; 3: 830-8.

59. Coppo R. Proteasome inhibitors in progressive renal diseases. Nephrol Dial Transplant. 2014; 29 Suppl 1: i25-i30.

60. Maurits E, van de Graaff MJ, Maiorana S, Wander DPA, Dekker PM, van der Zanden SY, et al. Immunoproteasome Inhibitor-Doxorubicin Conjugates Target Multiple Myeloma Cells and Release Doxorubicin upon Low-Dose Photon Irradiation. J Am Chem Soc. 2020; 142: 7250-3

61. Bhattarai D, Lee MJ, Baek A, Yeo IJ, Miller Z, Baek YM, et al. LMP2 Inhibitors as a Potential Treatment for Alzheimer's Disease. J Med Chem. 2020; 63: 3763-83.

62. Bockstahler M, Fischer A, Goetzke CC, Neumaier HL, Sauter M, Kespohl M, et al. Heart-Specific Immune Responses in an Animal Model of Autoimmune-Related Myocarditis Mitigated by an Immunoproteasome Inhibitor and Genetic Ablation. Circulation. 2020; 141: 1885-902.

63. Moallemian R, Rehman AU, Zhao N, Wang H, Chen H, Lin G, et al. Immunoproteasome inhibitor DPLG3 attenuates experimental colitis by restraining NF-KB activation. Biochem Pharmacol. 2020; 177: 113964.

64. Kuhn DJ, Hunsucker SA, Chen Q, Voorhees PM, Orlowski M, Orlowski RZ. Targeted inhibition of the immunoproteasome is a potent strategy against models of multiple myeloma that overcomes resistance to conventional drugs and nonspecific proteasome inhibitors. Blood. 2009; 113: 4667-76.

65. Niewerth D, Kaspers GJ, Assaraf YG, van Meerloo J, Kirk CJ, Anderl J, et al. Interferon- $\gamma$-induced upregulation of immunoproteasome subunit assembly overcomes bortezomib resistance in human hematological cell lines. J Hematol Oncol. 2014; 7 : 7

66. Zerfas BL, Trader DJ. Monitoring the Immunoproteasome in Live Cells Using an Activity-Based Peptide-Peptoid Hybrid Probe. J Am Chem Soc. 2019; 141: 5252-60.

67. Huber EM, Basler M, Schwab R, Heinemeyer W, Kirk CJ, Groettrup M, et al. Immuno- and constitutive proteasome crystal structures reveal differences in substrate and inhibitor specificity. Cell. 2012; 148: 727-38. 